\title{
Reducing GPS Impreciseness by Odometer Sensor Reading to Improve Positioning Accuracy
}

\author{
Murat Bakırc1 ${ }^{1}$ \\ 0000-0003-2092-1168 \\ ${ }^{1}$ Department of Aerospace Engineering, Faculty of Aeronautics and Astronautics, Tarsus University, Mersin, 33400, Turkey
}

\begin{abstract}
Positioning accuracy is becoming more and more important as autonomous vehicle technology develops. The focus of this paper is on an onboard smart mobile device's feature to improve positioning accuracy, based on experimentally acquired GPS and odometer sensor data. A simplificative odometer reading based approach is applied instead of using more advanced smart device sensors (e.g., accelerometer, gyroscope). Numerous driving tests were performed and analyzed to collect sufficient dynamic travel data. Traveled distances between two consecutive positions from GPS data are computed and correlated with a vehicle's speed profile between the same two positions. To calculate distance more precisely speed values from GPS are corrected with odometer sensor reading. The results revealed an average increase in accuracy of $20 \%$. The developed model can be incorporated with a smart device's other low-energy sensors. Using the smart device sensors, the developed model can be extended to acquire a more accurate positioning.
\end{abstract}

Keywords: Positioning; GPS inaccuracy; Smart mobile device; Odometer; Dynamic travel data; Intelligent transportation systems
Research Article

https://doi.org/10.30939/ijastech..977039

$\begin{array}{ll}\text { Received } & 31.07 .2021 \\ \text { Revised } & 11.09 .2021 \\ \text { Accepted } & 14.09 .2021\end{array}$

* Corresponding author

Murat Bakırcı

muratbakirci@tarsus.edu.tr

Address: Department of Aerospace Engineering, Faculty of Aeronautics and Astronautics, Tarsus University, Mersin, Turkey

Tel: +903246000060

\section{Introduction}

Because of its low cost, continuous and global solution, GPSbased positioning is widely used in almost every transportation systems. Although in recent years different techniques have been used to improve positioning accuracy [1-6], they are not sufficient enough to support operations that require high accuracy [7-14]. Due to enhancement of received signals from satellites, a better positioning can be obtained by multiple use of various Global Navigation Satellite Systems (GNSS) (e.g., GPS and GLONASS) [1517]. Through the use of multiple GNSSs, an elimination of residual range errors on both $\mathrm{L} 1$ and $\mathrm{L} 2$ ray paths can be made which provides more precise correction for the signal delay and phase advance induced by ionosphere [18]. However, new transportation applications such as automatic guide and control systems for driverless vehicles will require a higher level of positioning accuracy [19-20]. Because of this, precise positioning will play an important role in the functionality of future transportation systems.

The importance of precise positioning will be emphasized in urban area where a high level of positioning accuracy will be needed for proper integration. Unfortunately, the dense urban environment causes an inconsistency in reception of the GPSs' signals [7], [2122]. Because of environmental interferences the performance of the GPS is significantly decreased, which reduces the chance of a successful integration [23-24]. Obstacles in urban areas degrade the amount of signals from satellites and, thus, limit the optimal reachable performances significantly [25]. Additional considerations regarding signal interference are reflections. Reflections cause a certain amount of delay to signal. These delays can cause additional positioning errors which are result of non-Gaussian distribution of the pseudorange error [25-26]. Recent studies demonstrate a wide range of methods to improve positioning accuracy as shown in Table 1. With the use of techniques and tools such as Inertial Navigation System (INS), In-Vehicle Sensors (IVS), Camera, Distance Measuring Instruments (DMI), LiDAR, Statistical Methods (SM), and Image Processing (IP), researchers attempt to increase precision of vehicle positioning. In [1], the authors propose the adaptive GPS/INS integration method to reduce INSrelated errors and achieve more consistent positioning. Developing two new filtering techniques, they estimate the covariances of GPS and INS measurements separately with these filters. A new twostage method to improve positioning accuracy is given in [2]. 
Table 1. Comparison of methodologies.

\begin{tabular}{l|c|c|c|c|c|c|c|c|c}
\hline \multicolumn{1}{c|}{ Research } & GNSS & INS & IVS & Cam & DMI & LiDAR & SM & IP & OBD \\
\hline Cai et al. 2018 [4] & + & & & + & & & & & \\
\hline Gakne et al. 2018 [3] & + & & & + & & & & & \\
\hline Karaim 2019 [20] & + & + & & & & & & & \\
\hline Lee et al. 2016 [1] & + & + & & & & & & & \\
\hline Marais et al. 2012 [7] & + & & & & & & + & & \\
\hline Marais et al. 2013 [15] & + & & & & & & & + & \\
\hline Meng et al. 2017 [6] & + & + & & & + & + & & & \\
\hline Xu et al. 2018 [2] & + & + & + & & & & & & \\
\hline Presented Research & + & & & & & & & & + \\
\hline
\end{tabular}

In this study, an algorithm that adopts to random INS noise is developed as well as a predictor has also been developed that predicts INS errors during GPS outages. [3] and [4] use cameras with GPS to increase positioning accuracy. The method fuses an upward-facing camera system of a vehicle with GPS signals for navigation purposes in urban environments. Lidar technology with triple fusion of GPS, INS, and DMI has been used in [6]. To compensate for GPS jumps, the performance of the triple system has been increased by a multi-constraint fault detection method. In [7], the authors preferred statistical methods to reduce the positioning error. The vehicle route was reconstructed in 3D space with image processing and more consistent positioning information was obtained by matching the signal propagation information. A similar approach was also used in [15], but here the image processing technique was used much more intensively. Similar to [1], the fusion of GPS and INS is presented in [20]. The author has developed a tracking system architecture for a strongly coupled GPS/INS integrated system. This integrated system has increased the tracking robustness and sensitivity of the receivers, making the system less dependent on the INS. The proposed technique demonstrates that GPS inaccuracy can be reduced partially by a vehicle's odometer sensor reading through a smartphone applications to achieve a more precise position estimations. By introducing a practical and efficient solution which uses additional resources from current smartphone technology, this work extends the existing literature of studying positioning accuracy for ground transportation systems. The method presented here, unlike those in the literature, corrects GPS-related positioning errors using the vehicle's odometer data. Utilizing a mobile application that works with Android smart mobile devices, the vehicle's odometer data is collected, which is much more consistent than the speed information provided by GPS. Inconsistent speed profiles obtained from GPS are corrected with this data, and more accurate positioning information is inferred from the corrected speed profiles.

\section{Methodology}

An Android smartphone, which is embedded with on-board low-energy sensors (e.g., gyroscope, accelerometer, and compass), have been installed with a custom Android application that provides beneficial information so it can collect GPS and vehicular sensor data for modeling and analysis. In addition to collecting GPS data (latitude, longitude, speed, etc.), the vehicle's odometer data was also collected through a backend that directly communicates with in-vehicle sensors, for this particular study. Furthermore, fuel consumption data, rpm information, and other pertinent information can also be collected from the moving vehicle by On-Board Diagnostic (OBD) sensor [27-28]. Although similar studies with smartphones encompass the whole range of data processing activities including code development [29-31], modeling and simulation [32-34], implementation, analysis and visualization [34]; collecting these data through smartphone from a vehicle requires no proficiency. The route used for the experiments consisted of bridges, skyscrapers, and tall trees which can cause interference by blocking GPS signals which may reduce localization accuracy to a certain extent.

The primary process for this methodology is to obtain the nearest correlation between vehicle speed and distance traveled for every two consecutive data points. In other words, the speed profile is to be matched with the distance profile of the vehicle. This may be a reasonable expectation since there exists a linear relationship between the two. However, this is not always true as there is a non-linear relationship when sudden speed variations happen (In the case of sudden braking, a big speed change occurs while distance change becomes relatively smaller). In addition, GPS response is not accurate as in moderate speed variations. As in most cases, large amount of data could be double-edged sword if not properly processed [7], [35-36]. Therefore, the collected data set must be divided into two subsets as direct and indirect correlated parts and each subset must be considered independently. When there exists a non-linear relationship, correlation ratio, $\eta$, which is a coefficient of non-linear associations arises. The model proposed in this paper was applied to direct correlated data only.

It is assumed that the vehicle travels through as straight path between two data points and, thus, Euclidean distances are computed between the points. The Euclidean distance between two points in the plane with coordinates $(u, v)$ is the length of the line segment 
connecting them as shown below.

$$
d=\|u-v\|
$$

Considering each points in the data set, Euclidean space $\left(\mathbb{R}^{n}\right)$ becomes a metric space, also called Euclidean distance matrix $d_{i, j}$, which is a set possessing a metric as shown in the expression below.

$$
d_{i j}=\left\|x_{i}-x_{j}\right\|_{2}^{2} \triangleq\left(x_{i}-x_{j}, x_{i}-x_{j}\right)
$$

Since it is symmetrical and all diagonal elements are zero, matrix $d_{i, j}$ has $i^{2}$ (or $j^{2}$ ) entries but only $i(i-1) / 2$ pieces of information. This is reasonable since the speed limit on the traveled route can vary between $25 \mathrm{mph}$ and $40 \mathrm{mph}$.

Next, the speed profile is overlapped with the computed distance profile as they are directly proportional. This could be performed by either statistical methods [35-36] or normalization which is more practical way. Both methods were applied however normalization method is preferred for the rest of the work. Speed profile and corresponding Euclidean distance matrix were normalized to common scale. Normalized form of a distance matrix $d_{i, j}$ is given by scaling features that lie between a given minimum $(\delta)$ and maximum $(\phi)$ value as shown in Eq. (3).

$$
\left[d_{i j}\right]=\delta+\left|\frac{\left(\left\{d_{i j}-\left[d_{i j}\right]_{\min }\right\}\{\delta-\phi\}\right)}{\left(\left[d_{i j}\right]^{\text {max }}-\left[d_{i j}\right]_{\min }\right)}\right|
$$

In fact, this is not only important when comparing measurements that have different units, but it is also a general requirement for many machine learning applications [37]. Afterwards, dimensionless speed profile of the vehicle is corrected with dimensionless form of odometer data which is much more precise than GPS- sourced speed data. This can simply be done by replacing the speed profile obtained from GPS with odometer sensor reading. However, speed differences between the two data are also computed for a deeper investigation. After obtaining the corrected dimensionless speed profile, the corrected dimensionless distance profile can finally be found by combining with root mean square error (RMSE). This error will be used as a measure of spread of the distance values about the corrected distance profile. In the last step of the process dimensionless distance values were converted to dimensional form.

\section{Implementation}

Satellite, map and schematic views of the route used in the experiments are shown in Figure 1. Since the speed limit on the route used in the experiments was $45 \mathrm{mph}(72 \mathrm{~km} / \mathrm{h})$, the maximum speed of the vehicle was not above this limit during the trials. Experiments have been carried out only in the city, thus, it has not been tested what kind of result will be obtained at speeds above this limit. A 2008 Ford Explorer was used for the travel experiments. The trips were held between the east entrance of the Old Dominion University's main campus and the MacArthur Mall in the city center. The average distance between the start and finish is about 3,4 miles $(5,5 \mathrm{~km})$, which takes about 18 minutes in average traffic conditions and about 29 minutes in heavy traffic conditions. Before driving began, the smartphone was fixed in the utility area between the front seats so that it would not move during the test. Since the forward axis of the smartphone must coincide with the forward axis of the vehicle, this was also taken care of when fixing. Experiments were mostly carried out during working hours, that is, during peak traffic hours, the reason for choosing this time interval is that the experiments were intended to simulate a normal daily life driving.
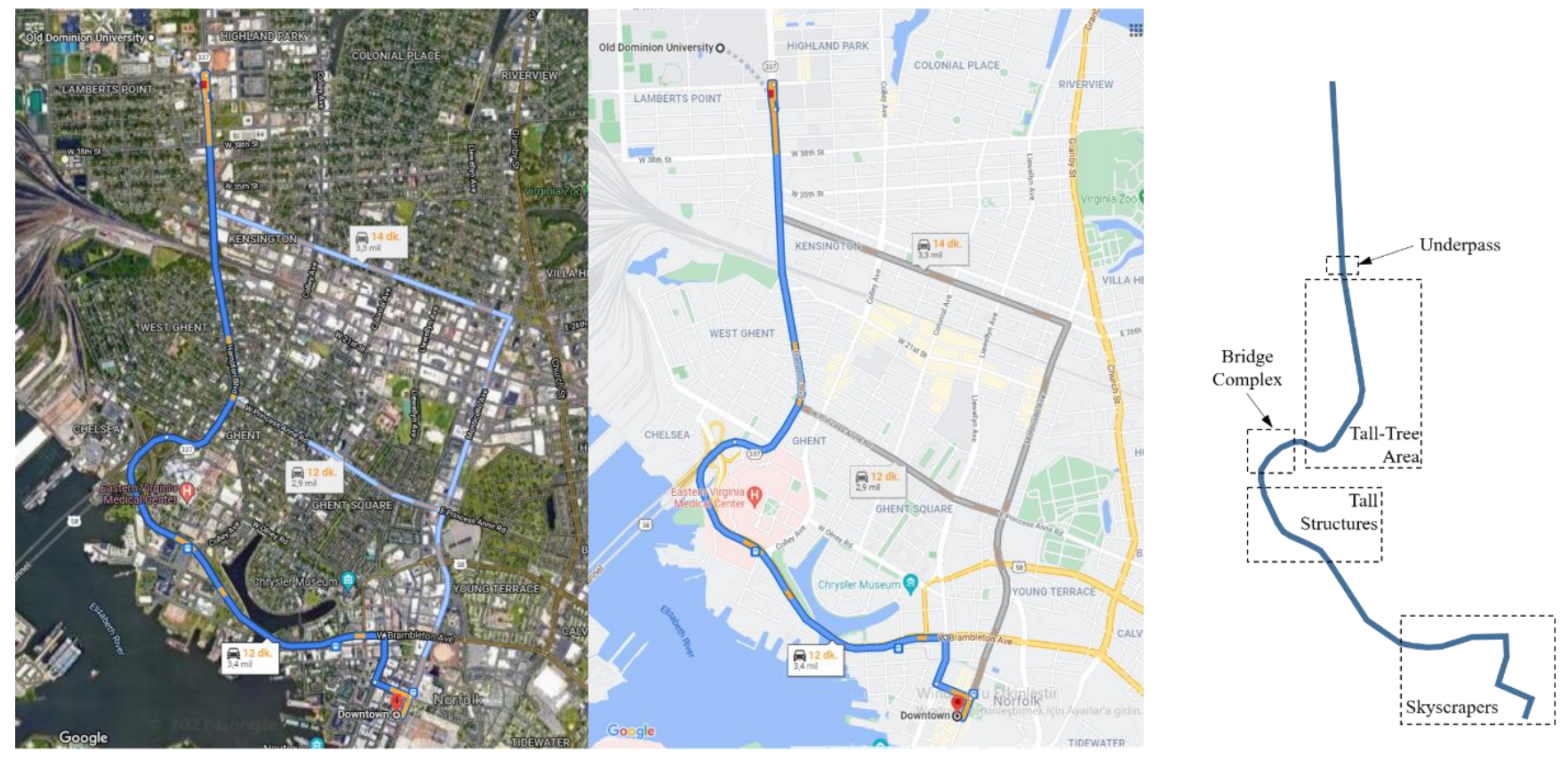

Fig. 1. Satellite, map and schematic views of the route used in the experiments. 


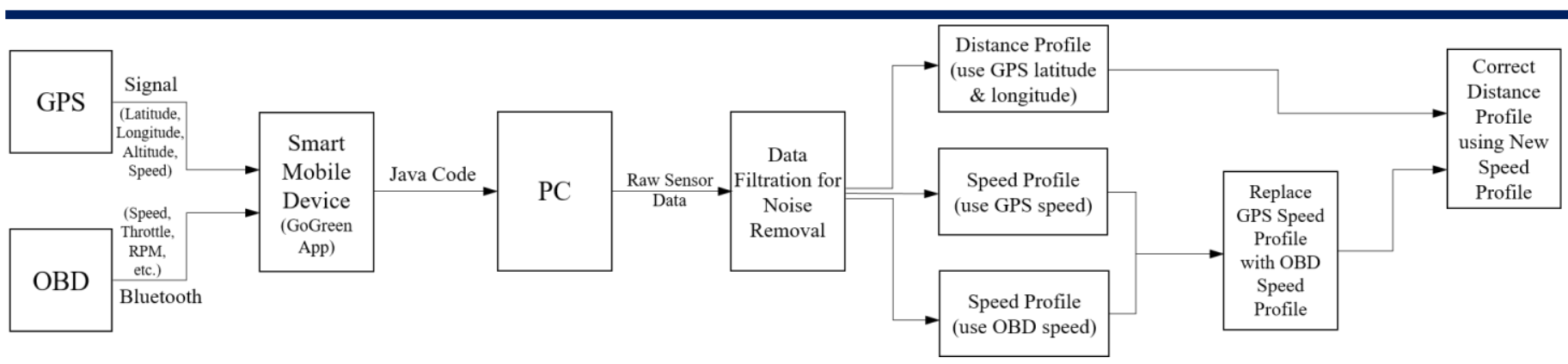

Fig. 2. Flowchart showing the steps of data collection and processing.

The GoGreen application developed by Transportation Research Institute of Old Dominion University was used to collect dynamic travel data with a smartphone. This application, particularly developed for Android smart mobile devices, has the ability to collect data from Micro-electro-mechanical-system (MEMS) sensors and GPS chips embedded in smart devices, as well as OnBoard Diagnostics (OBD) device data that transmits data to the smart mobile device via Bluetooth. When the application is activated with all its features, it can collect data measured by MEMS sensors, and also latitude, longitude, speed, time stamp information via GPS, as well as speed and throttle position data via OBD device. Once the application is stopped, it will prompt the user to upload the data into the database so that all readings can be saved to smart mobile device's local database. When uploading is finished, the mobile device is connected to a computer via USB cable and all dynamic travel data can be retrieved using a code written in Java.

In order to get OBD data, the data acquisition module of the application establishes a Bluetooth connection between the mobile device and the OBD, and sends a series of queries to the OBD to configure this connection. Once the connection is established successfully, it sends queries in a similar way and retrieves the realtime vehicle data at a specified rate. The stages of data collection and processing method are shown in the flowchart given in Figure 2.

The type of measurement utilized in high-precision applications for purposes of ensuring that accuracy is achieved irrespective of have very low code measurements is the phase measurements. Due to low quality of GPS chips embedded in smart devices and poor multipath mitigation, the mean error (RMS) on the code is approximately five meters and this results in a precision of approximately ten to fifteen meters in 3D. However, phase measurements produce much higher quality results than this. In addition to being preferred in many smart mobile device applications recently, phase measurements are thus utilized in studies focusing on Earth rotation, in approximation of troposphere with the aim of attaining high quality observations as well as geodetic topics such as global reference to frame realization. Although an application programming interface called GNSS Logger was added to Android operating systems with the Android 7 version, the custom-developed application GoGreen was preferred for this study. GoGreen stores the collected GPS observation data in ASCII output files. Then, data such as epoch time, code, carrier phase from these files are brought into readable mode via a converter. An important advantage of using this developed code instead of commercial ones is that it provides to know all stages of data processing. Moreover, each step can also be controlled if the measured data are processed with stochastic models, and this indeed helps to get more reliable results. Since GPS signals are collected inside a moving vehicle, they are subject to signal interferences and signal reflections from time to time, which causes a larger positioning error in the measurements compared to static observations. Thus, code and carrier phase observations are also less consistent in moving, i.e., kinematic situations, and therefore positioning involves more errors. It should also be noted that the antenna characteristics of smart devices are quite different from those of geodetic antennas and this causes a disadvantage in antenna gain. Even the fact that the antenna reference center does not coincide with the center of the smart device causes at centimeter level error in measurement accuracy. Moreover, the smart mobile device used in the experiments contains a single-frequency GPS receiver. This prevents ionospheric monitoring, unlike mobile devices using dual-frequency.

\section{Results}

An extensive data collection process has been performed in the study area during the calendar year 2019 and 2020 to test the proposed method. The speed profiles of the data sets were examined. The data sets with less zero-speed values were selected to be studied as this is an indication that the vehicle is in motion. For the remainder of this section, 'data' refers to these selected data sets. This is a reasonable approach as the proposed method does not make any contributions in case of vehicle stops, which effects time efficiency.

In addition to the speed profiles, distance matrices were computed for the data as well as corresponding distance profiles. These produce one sample of a distance profile as shown in Figure 3a. The plot represents the data's first thousand seconds. A proper data selection is inferred as the results show the vehicle is in motion approximately $81 \%$ of the time. The linearly correlated sections were identified and a random sample of 100 second was taken from these sections. The selected 100 seconds interval is between $t=318$ to $t=417$ an outlined in red as shown in Figure 3a. This sample will be referred as 'test data' and can be seen in Figure $3 \mathrm{~b}$. 

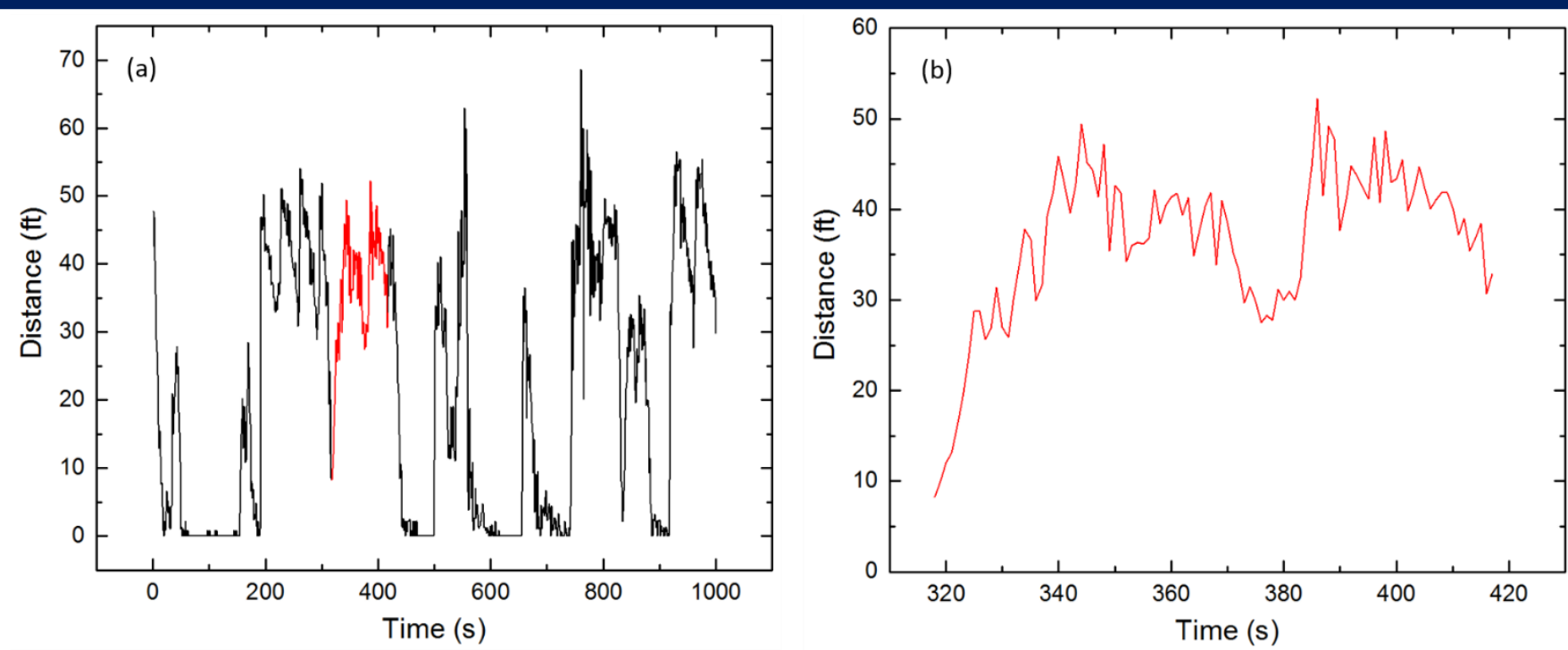

Fig. 3. A sample of distance profile (a) and the 'test data' (b).

The distance profile of the test data is linearly correlated with the corresponding speed profile of the vehicle. To verify this linearity, speed ratios between consecutive points were computed. These results can be seen in Figure 4 below.

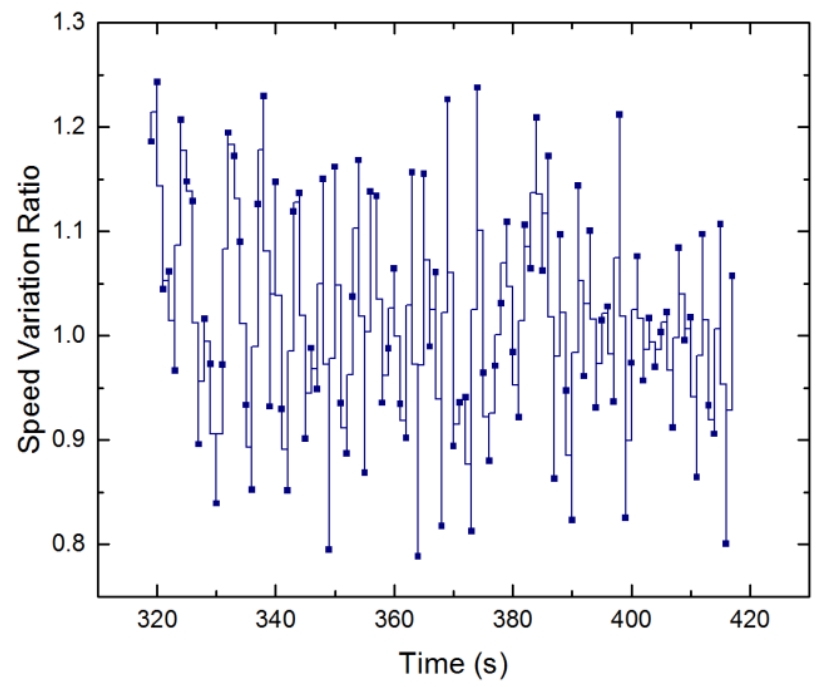

Fig. 4. Speed variation ratios between consecutive points.

When comparing decrement or increment in speed between consecutive points, it is hypothesized that speed change is not sudden when this value remains below $20 \%$. Within $93 \%$ confidence, test data vehicle speed does not vary more than $20 \%$ between consecutive points. This provides a confidence as shown in the plot (Figure 2). A correlation of the test data between speed and distance profiles was also computed. The result shown on the next page (Figure 5) bolster the hypothesis as their Pearson correlation coefficient is 0.97 , this demonstrates a high assurance of proper data selection.
To show each profile clearly, the test data and corresponding speed profiles were plotted as a dual-scaled axes (Figure 6a). With normalized data, as shown in Figure 6b, it can be seen that the two profiles overlap to a great extent. A RMSE of 0.038 and a mean absolute error (MAE) of 0.033 were computed as an indicator for prediction error between the two profiles. These errors will be used as a measure of spread of the distance values about the corrected distance profile.

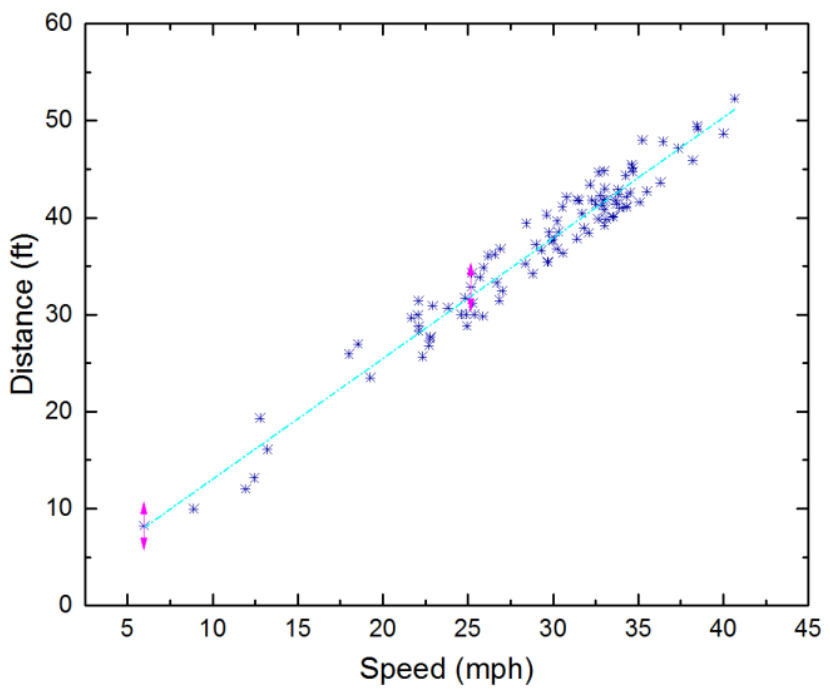

Fig. 5. Correlation between speed and distance profiles.

The plot shown in Figure 7a represents the difference between GPS-sourced and odometer-based speed profiles corresponding to the test data. This figure shows the average speed difference is 2.79 $\mathrm{mph}$ and that $42 \%$ of the time the difference is larger than $3 \mathrm{mph}$. The normalized version of the speed difference can also be seen below (Figure 7b). By adding the normalized speed differences to 

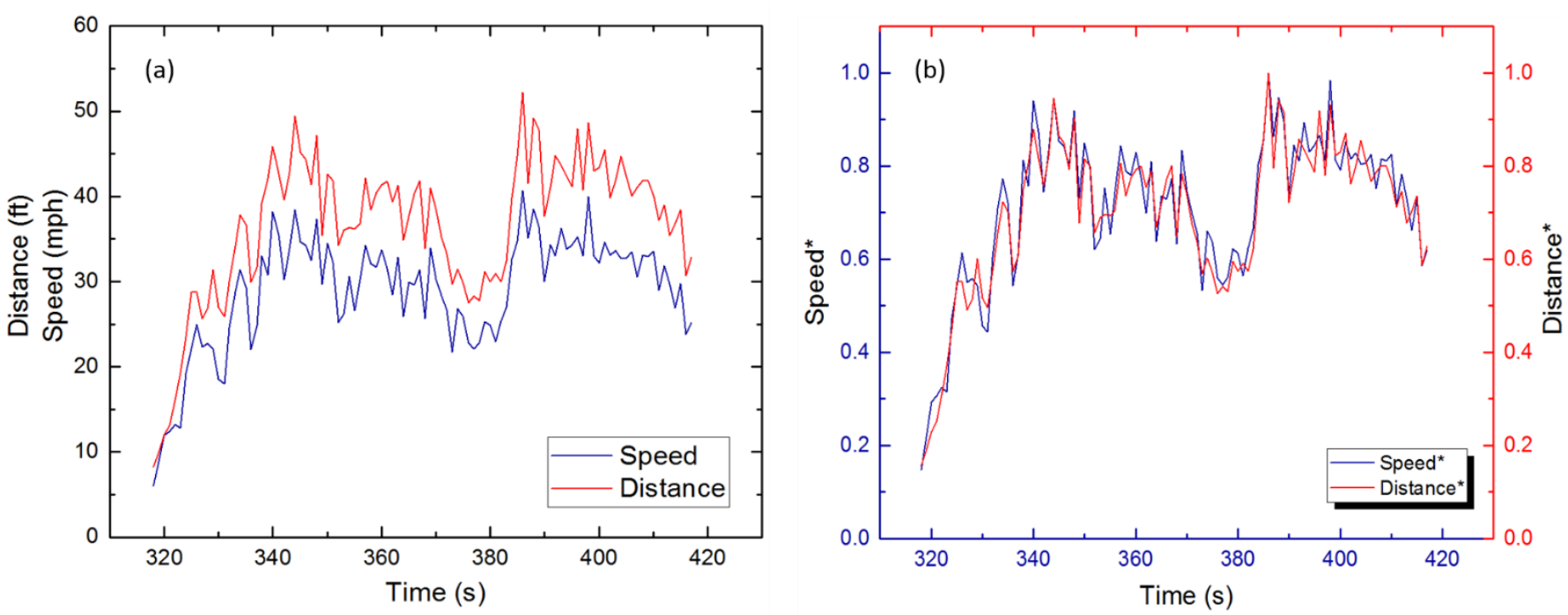

Fig. 6. Speed and distance profiles (a), and normalized form (b).
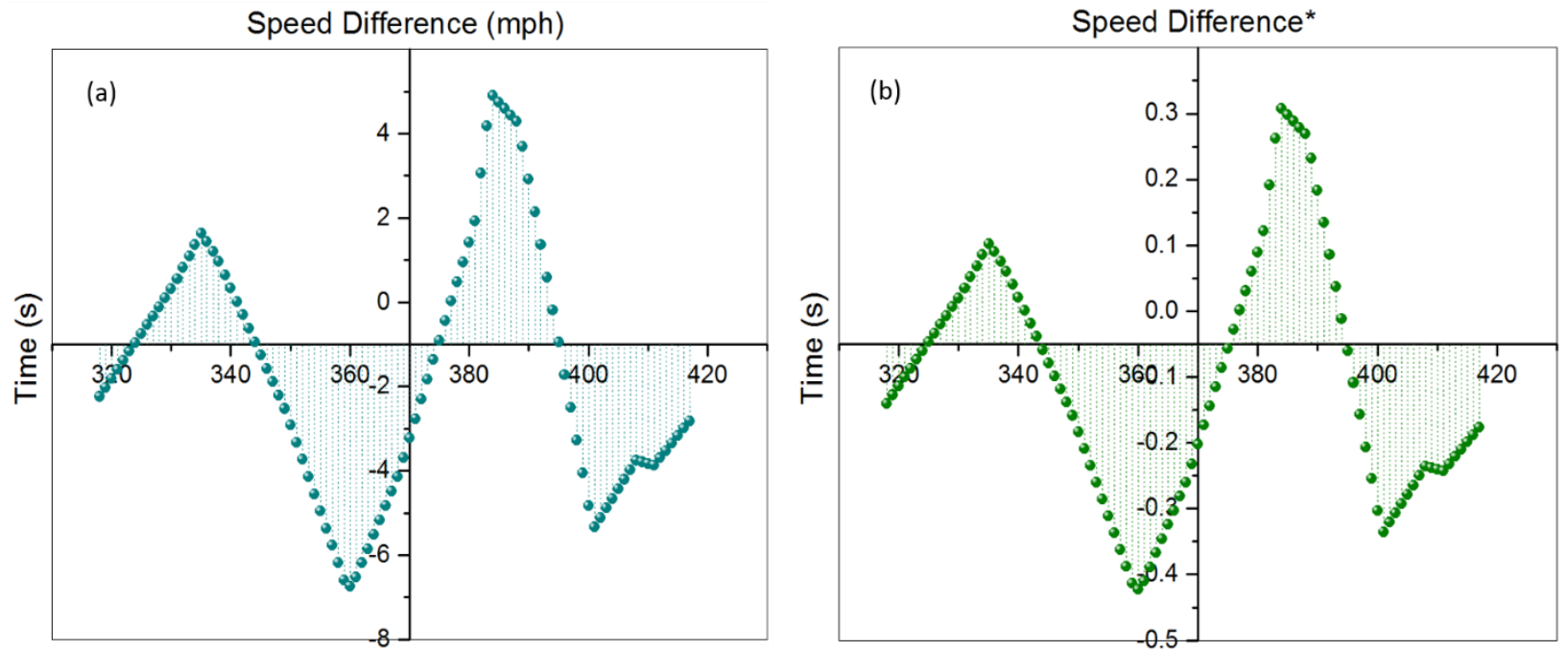

Fig. 7. Speed difference between GPS-sourced and odometer-based speed profiles (a) and normalized form (b).

the normalized speed profile, the corrected speed profile is obtained as shown in Figure 8a. Significant distinction can clearly be seen between $t=347$ and $t=373 ; t=381$ and $t=$ $391 ; t=396$ and $t=417$. Since RMSE is larger than MAE, it is preferred for spreading to speed values about the speed profile to obtain corrected distance profiles as shown in Figure $8 \mathrm{~b}$.

In the graph shown on the next page (Figure 9a), normalized distance and corrected normalized distance profiles are plotted. Finally, the plot (Figure 9b) shows a corresponding dimensional graph that resulted in correction of distance with an average of 9.21 $\mathrm{ft}$ and maximum of $23.15 \mathrm{ft}$.

\section{Conclusions and Future Work}

A smartphone's feature to improve positioning accuracy for ground transportation systems is surveyed in this paper. The study was aimed to reduce GPS-sourced positioning error by processing large amount of dynamic travel data collected by smartphones. The proposed model is concentrated on generating the nearest correlation between speed and distance profiles of vehicle. The work advances the existing literature by presenting a practical and efficient solution while making minimal use of low-energy sensors of smartphones. The model validation was made by applying to variety of collected test data which consist no sudden speed variations. An average of $11.3 \mathrm{ft}$ which corresponds to approximately $20 \%$ correction in distance was achieved. However, the model demonstrated in this paper should also be tested with the data which involve sudden speed variations. Using the corrected distance profiles, a procedure may be developed to update GPS-sourced positioning instantaneously. The work may be advanced using a camera application while collecting dynamic travel data to make a 

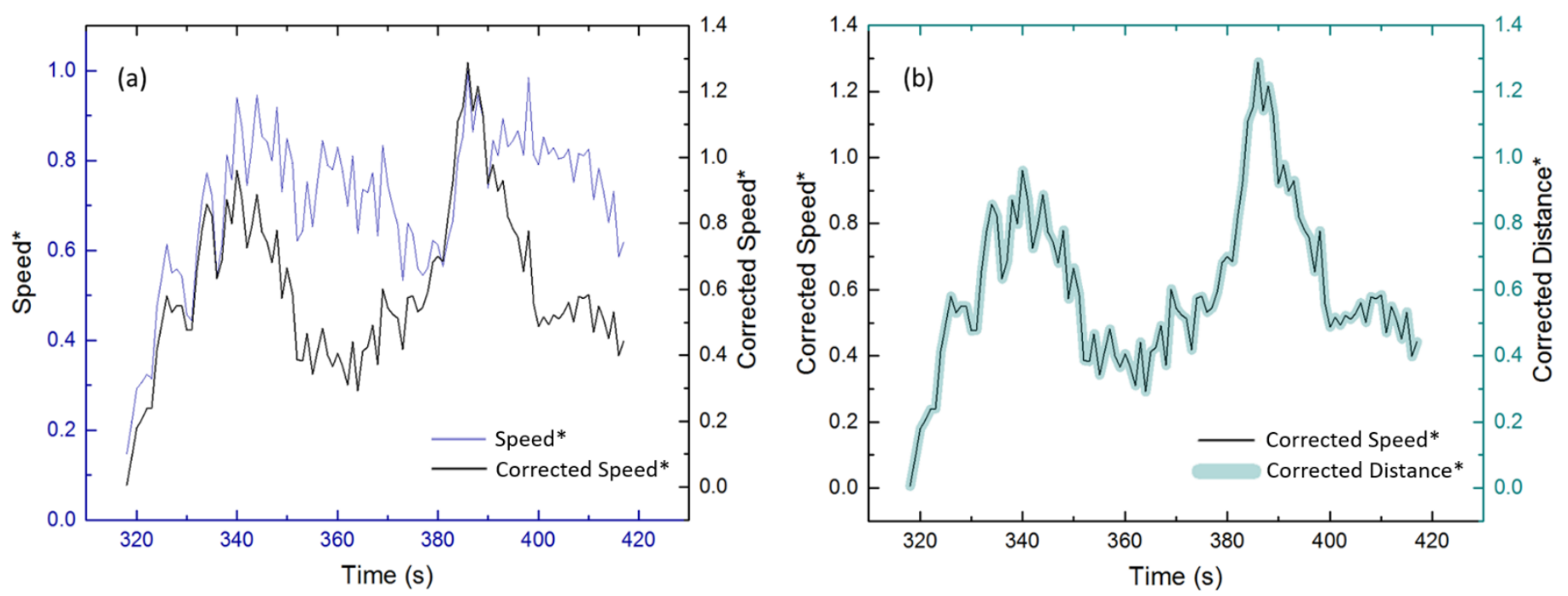

Fig. 8. Corrected speed profile (a) and corrected distance profile (b).
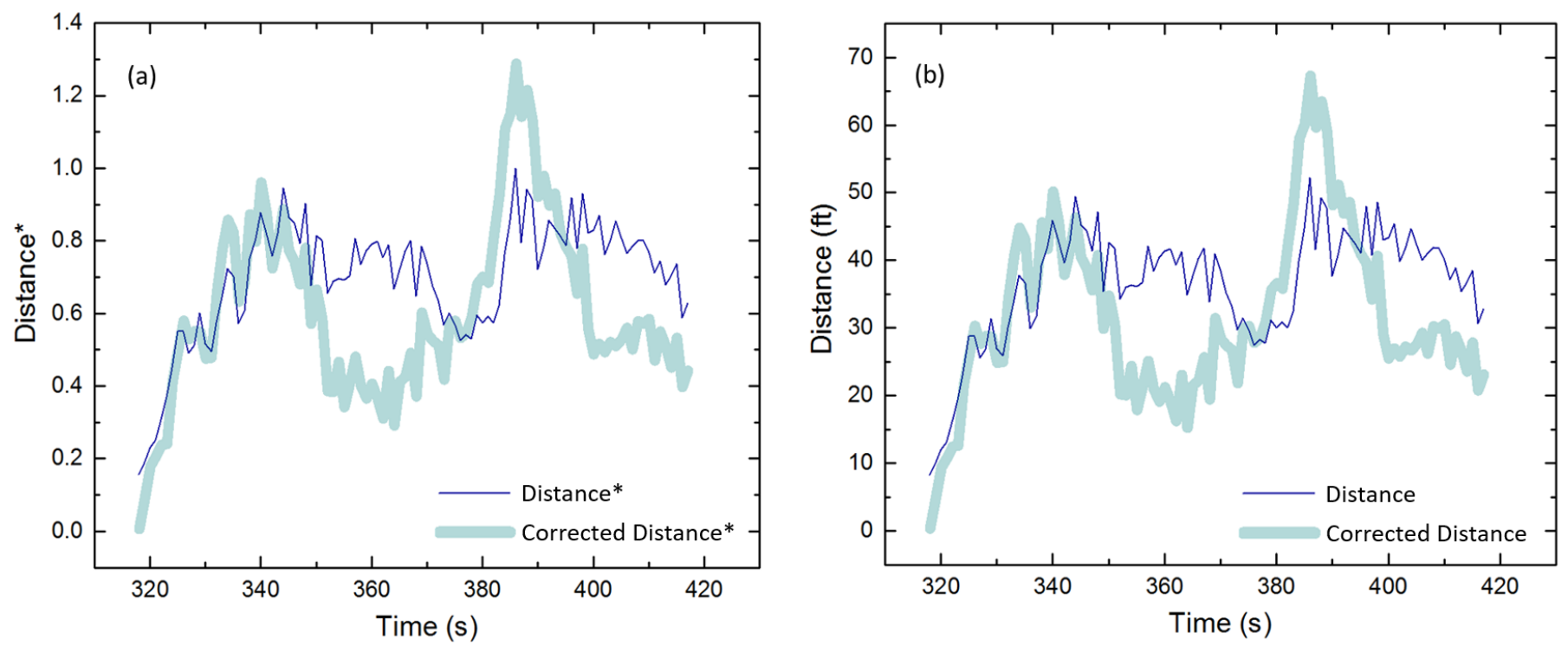

Fig. 9. Normalized (a) and dimensional form (b) of corrected distance profile.

deeper investigation on positioning error sources. The data used in proposed model may also be incorporated with other low-energy sensor data to further enhance the model discussed in this paper.

\section{Acknowledgment}

This study was partially supported by Transportation Research Institute (TRI) of Old Dominion University, Norfolk, VA. As the author, I am grateful for the contributions of TRI.

\section{Nomenclature}

$u \quad$ : any location in the $2 \mathrm{D}$ plane

$v \quad:$ any location in the $2 \mathrm{D}$ plane $d \quad$ : Euclidean distance between any two points (ft)

$d_{i, j} \quad:$ Euclidean distance matrix

$x_{i} \quad:$ any point in the $2 \mathrm{D}$ plane

$x_{j} \quad$ : any point in the 2D plane

$\delta \quad:$ the element with the lowest value of the norm alized distance matrix

$\phi \quad$ : the element with the highest value of the norm alized distance matrix

\section{Conflict of Interest Statement}

The author declares that there is no conflict of interest. 


\section{References}

[1] Lee JY, Kim HS, Choi KH, Lim J, Chun S, Lee HK. Adaptive GPS/INS integration for relative navigation. GPS Solutions. 2016;20(1):63-75.

[2] Xu Q, Li X, Chan CY. Enhancing localization accuracy of MEMSINS/GPS/In-vehicle sensors integration during GPS outages. IEEE Transactions of Instrumentation and Measurement. 2018;67(8):1966-1978.

[3] Gakne PV, O'Keefe K. Tightly-coupled GNSS/vision using a skypointing camera for vehicle navigation in urban areas. Sensors. 2018;18(4):1244.

[4] Cai H, Hu Z, Huang G, Zhu D, Su X. Integration of GPS, monocular vision, and high definition (HD) map for accurate vehicle localization. Sensors. 2018;18(10):3270.

[5] Drawil NM, Amar HM, Basir OA. GPS localization accuracy classification: a context-based approach. IEEE Transactions on Intelligent Transportation Systems. 2013;14(1):262-273.

[6] Meng X, Wang H, Liu B. A robust vehicle localization approach based on GNSS/IMU/ DMI/LiDAR sensor fusion for autonomous vehicles. Sensors. 2017;17(9):2140.

[7] Marais J, Ambellouis S, Flancquart A, Lefebvre S, Meurie C, Ruichek Y. Accurate localization based on GNSS and propagation knowledge for safe applications in guided transport. Social and Behavioral Sciences. 2012;48:796-805.

[8] Li X, Guo J, Zhang D. An algorithm of GPS single-epoch kinematic positioning based on doppler velocimetry. Geomatics and Information Science of Wuhan University. 2018;43:1036-1041.

[9] Ge Y, Dai P, Qin W, Yang X, Zhou F, Wang S, Zhao X. Performance of multi-GNSS precise point positioning time and frequency transfer with clock modeling. Remote Sensing. 2019;11:347.

[10]Yu X, Gao J. Kinematic precise point positioning using multi-constellation global navigation satellite system (GNSS) observations. ISPRS International Journal of Geo-Information. 2017;6(6):1-15.

[11]Cristorado C, Falco G, Ruotsalainen L, Dovis F. On the use of an ultra-tight integration for robust navigation in jammed scenarios. 32nd International Technical Meeting of the Satellite Division of The Institute of Navigation, Miami, Florida, USA, 2019.

[12]Jiang H, Hu J, An S, Wang M, Park BB. Eco approaching at an isolated signalized intersection under partially connected and automated vehicles environment. Transportation Research Part C: Emerging Technologies. 2017;79:290-307.

[13]Patel RH, Harri J, Bonnet C. Impact of localization errors on automated vehicle control strategies. IEEE Vehicular Networking Conference. Italy, 2017;61-68.

[14]Li Y, Hu ZZ, Hu YZ, Chu D. Integration of vision and topological self-localization for intelligent vehicles. Mechatronics. 2018;51:4658.

[15]Marais J, Meurie C, Attia D, Ruichek Y, Flancquart A. Toward accurate localization in guided transport: combining GNSS data and imaging information. Transportation Research Part C: Emerging Technologies. 2013; <hal-00879677>.

[16]Sekaran J, Kaluvan H, Irudhayaraj L. Modeling and analysis of GPSGLONASS navigation for car like mobile robot. Journal of Electrical Engineering Technology. 2020;15:927-935.
[17]Hsu L, Gu Y, Kamijo S. 3D building model-based pedestrian positioning method using GPS/ GLONASS/QZSS and its reliability calculation. GPS Solutions. 2016;20:413-428.

[18]Ioannides RT, Strangeways HJ. Improved ionospheric correction for dual frequency GPS. Department of Electronics and Electrical Engineering, The University of Leeds LS2 9JT, UK.

[19]Keicher R, Seufert H. Automatic guidance for agricultural vehicles in Europe. Computers and Electronics in Agriculture. 2000;25:169194.

[20]Karaim M. Ultra-tight GPS/INS integrated systems for land vehicle navigation in challenging environments. Dissertation. Queen's University, 2019.

[21]Krach B, Lentmaier M, Robertson P. Joint Bayesian positioning and multipath migitation in GNSS. IEEE. 2008;DOI:10.1109/ICASSP.2008.4518390:3437-3440.

[22]Ng Y, Gao GX. GNSS multireceiver vector tracking. IEEE Transactions on Aerospace and Electronic Systems. 2017;53:2583-2593.

[23]Brida P, Machaj J, Benikovsky J. A modular localization system as a positioning service for road transport. Sensors. 2014;14:2027420296.

[24]Schmidt GT. GPS based navigation systems in difficult environments. Gyroscopy and Navigation. 2019;10:41-53.

[25]Fan J, Ma G. Characteristics of GPS positioning error with non-uniform pseudorange error. GPS Solutions. 2014;18(4):615-623.

[26]Dautermann T, Mayer C, Antreich F, Konovaltsev A, Belabbas B, Kalberer U. Non-Gausian error modeling for GBAS integrity assesment. IEEE Transactions on Aerospace and Electronics Systems. 2012;48(1):1-14.

[27]Lin CE, Li CC, Yang SH, Lin SH, Lin CY. Development of on-line diagnostic and real time early warning system for vehicles. Sensors for Industry Conference. Houston, Texas, USA, 2005.

[28]Li R, Wang S, Long Z, Gu D. Undeepvo: monocular visual odometry through unsupervised deep learning. IEEE International Conference on Robotics and Automation. Australia, 2018;7286-7291.

[29]Berger M, Platzer M. Field evaluation of the smartphone-based travel behaviour data collection app "SmartMo". 10 $10^{\text {th }}$ International Conference on Transport Survey Methods, Transportation Research Procedia. 2015;11:263-279.

[30]Castrogiovanni P, Fadda E, Perboli G, Rizzo A. Smartphone data classification technique for detecting the usage of public or private transportation modes. IEEE Access. 2020;8:58377-58391.

[31]Mukheja P, Velaga NR, Sharmila RB. Smartphone-based crowdsourcing for position estimation of public transport vehicles. IET Intelligent Transport Systems. 2017;11(9):588-595.

[32] Yang F, Chen L, Cheng Y, Luo X, Ran B. An empirical study of parameter estimation for stated preference experimental design. Mathematical Problems in Engineering. 2014;2014: ID 292608, http://dx.doi.org/10.1155/2014/292608.

[33]Talebpour A, Mahmassani HS, Bustamante FE. Modeling driver behavior in a connected environment: integrated microscopic simulation of traffic and mobile wireless telecommunication systems. Transportation Research Record. 2016;2560(1):75-86.

[34] Ma X, Wu YJ, Wang Y. E-science tansportation platform for data sharing, visualization, modeling, and analysis. Transportation Research Record: Journal of the Transporttion Research Board. 
2011;2215:37-49.

[35]Karlaftis MG, Vlahogianni EI. Statistical methods versus neural networks in transportation research: differences, similarities and some insights. Transportation Research Part C. 2011;19:387-399.

[36]Piasco N, Sidibe D, Demonceaux C, Gouet-Brunet V. A survey on visual based localization: on the benefit of heterogeneous data. Pattern Analysis and Applications. 2018;74:90-109.

[37]Antoniou C, Koutsopoulos HN. Estimation of traffic dynamics models with machine-learning methods. Transportation Research Record: Journal of the Transporttion Research Board. 2006;1965:103- 